\title{
Contribution of an interharmonic component to the sine- wave parameters estimators returned by the interpolated Discrete Fourier transform algorithm
}

\author{
Daniel Belega ${ }^{1}$, Dario Petri², Dominique Dallet ${ }^{3}$ \\ ${ }^{1}$ Department of Measurements and Optical Electronics, University Politehnica of Timișoara, Bv. V.Pârvan, Nr. 2, 300223, Timișoara, \\ Romania \\ ${ }^{2}$ Department of Industrial Engineering, University of Trento, Via Sommarive, 9, 38123, Trento, Italy \\ ${ }_{3}^{3}$ IMS Laboratory, Bordeaux INP, University of Bordeaux, CNRS UMR5218, 351 Cours de la Libération, Bâtiment A31, 33405, Talence Cedex, \\ France
}

\begin{abstract}
This article investigates the contribution of a small-amplitude interharmonic component to the sine-wave parameter estimators returned by the classical interpolated discrete Fourier transform (IpDFT) algorithm. The analytical expressions for the frequency, amplitude, and phase estimation errors are derived herein by considering the IpDFT algorithm based on the maximum sidelobe decay (MSD) windows and by assuming the interharmonic frequency located at least one bin apart the unknown sine-wave frequency. The derived expressions allow us to analyse the impact of an interharmonic on the accuracies of the IpDFT frequency, amplitude, and phase estimators. The accuracies of the derived expressions are verified by means of both computer simulations and experimental results.
\end{abstract}

\section{Section: RESEARCH PAPER}

Keywords: error analysis; interharmonic component; interpolated DFT algorithm; parameter estimation; windowing

Citation: Daniel Belega, Dario Petri, Dominique Dallet, Contribution of an interharmonic component to the sine-wave parameters estimators returned by the interpolated DFT algorithm, Acta IMEKO, vol. 9, no. 3, article 4, September 2020, identifier: IMEKO-ACTA-09 (2020)-03-04

Editor: Jan Saliga, Technical University of Košice, Slovakia

Received January 15, 2020; In final form April 1, 2020; Published September 2020

Copyright: This is an open-access article distributed under the terms of the Creative Commons Attribution 3.0 License, which permits unrestricted use, distribution, and reproduction in any medium, provided the original author and source are credited.

Corresponding author: Daniel Belega, e-mail: daniel.belega@upt.ro

\section{INTRODUCTION}

Frequency-domain algorithms based on the discrete Fourier transform (DFT) return highly accurate sine-wave parameters estimates under coherent sampling conditions [1]. However, in practice, coherent sampling is often difficult to achieve, since the sine-wave frequency can be time-variant, and the waveform can be affected by spurious tones. Time-domain approaches, such as those implemented by the Prony, Pisarenko, and MUSIC algorithms [2], [3], also ensure accurate sine-wave parameter estimates when non-coherent sampling occurs. Unfortunately, the required processing effort is quite high, and the estimation accuracy depends on the order of the adopted waveform model, which is normally unknown a priori. Therefore, these approaches do not provide optimal performance in real-time applications, for which frequency domain algorithms are often preferred due to their lower processing effort. Unfortunately, when noncoherent sampling occurs, the sine-wave parameter estimates provided by DFT-based algorithms are affected by both spectral leakage [4] and picket-fence effects [5]. The spectral leakage effect can be significantly reduced by windowing [4], and cosineclass windows are frequently used, since they can be easily implemented and ensure a good spectral leakage suppression. Conversely, the picket-fence effect can be reduced by applying one of the so-called interpolated DFT algorithms [5]-[21]. The classical IpDFT algorithm [8] firstly estimates the sine-wave frequency by interpolating the two highest samples of the signal spectrum. Then, the sine-wave amplitude and phase are determined considering the signal DFT and the estimated frequency. When a small number of sine-wave cycles is acquired under non-coherent sampling conditions, the obtained parameter estimates are significantly affected by the spectral interference raising from the fundamental image component. The related contribution can be reduced by using more interpolation points [15]-[21], but at the expenses of an estimator variance increase [17]. IpDFT estimators are expressed by very simple expressions when maximum sidelobe decay (MSD) windows are employed [4], [22]. These are cosine windows that 
exhibit the maximum spectrum sidelobe decay rate for a given number of terms, thereby effectively reducing the detrimental effect on the estimated parameters of long-range spectral leakage due to harmonics or spurious tones. However, the effectiveness of windowing on short-range leakage is very low, such that the influence of nearby interfering tones on the estimated parameters can be significant. When the classical IpDFT algorithm based on an MSD window is applied, expressions for the frequency error and the amplitude and phase errors due to short-range interference from both the image component and harmonics have been derived in [23] and [24], respectively. However, reallife sine-waves are also affected by interharmonics. An expression for the frequency error affecting the IpDFT algorithm when the analysed sine-wave is corrupted by a smallamplitude interharmonic located at least one bit apart the unknown frequency has been derived in [25]. Our aim in this article is to extend that result by deriving the expressions for the amplitude and phase estimation errors that affect the classical IpDFT algorithm based on an MSD window. The derived expressions generalise the results related to the sine-wave affected by one harmonic. They allow us to easily analyse the contribution of an interharmonic on the amplitude and phase estimates returned by the IpDFT algorithm. To the best of the authors' knowledge, such an analysis has not yet been published. The aforementioned expressions are outlined in Section 2, where some remarks about the contribution due to a nearby interharmonic are also reported. The accuracies of the derived expressions and the correctness of the remarks are then verified by means of computer simulations in Section 3. Finally, conclusions are proposed in Section 4.

\section{SINE-WAVE AFFECTED BY AN INTERHARMONIC: IPDFT FREQUENCY, AMPLITUDE, AND PHASE ESTIMATION ERRORS}

The analysed discrete-time waveform is modelled as:

$$
\begin{aligned}
& x(m)=A \cos \left(2 \pi \frac{f_{0}}{f_{\mathrm{s}}}\left(m+\frac{1}{2}\right)+\phi\right) \\
& +A_{\mathrm{ih}} \cos \left(2 \pi \frac{f_{\mathrm{oih}}}{f_{\mathrm{s}}}\left(m+\frac{1}{2}\right)+\phi\right) \\
& m=-\frac{M}{2},-\frac{M}{2}+1, \ldots, \frac{M}{2}-1
\end{aligned}
$$

where $A, f_{0}, \phi, A_{\mathrm{ih}}, f_{0 \mathrm{ih}}$, and $\phi_{\mathrm{in}}$ are the amplitude, frequency, and initial phase of the fundamental and the interharmonic components, respectively, and $f_{\mathrm{s}}$ is the sampling frequency, while $M$ is the acquisition length.

The ratios $f_{0} / f_{\mathrm{s}}$ and $f_{\text {oih }} / f_{\mathrm{s}}$ can be expressed as:

$$
\begin{aligned}
& \frac{f_{0}}{f_{\mathrm{s}}}=\frac{v}{M}=\frac{l+\delta}{M}, \\
& \frac{f_{\mathrm{oih}}}{f_{\mathrm{s}}}=\frac{v_{\mathrm{ih}}}{M}=\frac{l_{\mathrm{ih}}+\delta_{\mathrm{ih}}}{M},
\end{aligned}
$$

where $v$ and $v_{\text {in }}$ are the number of acquired cycles; $l$ and $l_{\text {in }}$ are the integer parts of $v$ and $v_{\mathrm{h}}$, respectively. Finally, $\delta(-0.5 \leq \delta<$ $0.5)$ and $\delta_{\mathrm{hh}}\left(-0.5 \leq \delta_{\mathrm{hh}}<0.5\right)$ are the corresponding fractional parts, i.e. the inter-bin frequency locations.

It is noteworthy that the reference time (i.e. the instant $m=0$ ) is chosen in the centre of the observation interval so that the IpDFT phase estimator is independent of the frequency estimator, and more accurate estimate can be obtained [11], as it will be shown in the next section.
In practice, both the fundamental and the interharmonic components are normally non-coherently sampled (i.e. $\delta \neq 0$ and $\left.\delta_{\mathrm{h}} \neq 0\right)$, so the discrete spectrum of (1) is affected by spectral leakage. To mitigate the issue, the windowed signal $x_{\mathrm{w}}(m)=$ $x(m) \cdot w(m)$ is analysed, where $w(\cdot)$ is a suitable window function. An $H$-term MSD window $(H \geq 2)$ is considered due to its good spectral characteristics [4], [22]. It is defined as:

$$
\begin{aligned}
& w(m) \stackrel{\text { def }}{=} \sum_{h=0}^{H-1} a_{h} \cos \left(2 \pi \frac{h}{M}\left(m+\frac{1}{2}\right)\right), \\
& m=-\frac{M}{2},-\frac{M}{2}+1, \ldots, \frac{M}{2}-1
\end{aligned}
$$

where the window coefficients $a_{b}$ are given by [12]: $a_{0}=\frac{C_{2 H-2}^{H-1}}{2^{2 H-2}}, a_{h}=\frac{C_{2 H-2}^{H-h-1}}{2^{2 H-3}}, h=1,2, \ldots, H-1$, in which $C_{a}^{b}=$ $\frac{a !}{(a-b) ! b !}$

The DFT of the windowed signal $\chi_{\mathrm{w}}(\cdot)$ is given by:

$$
\begin{aligned}
& X_{w}(k) \stackrel{\text { def }}{=} \sum_{m=-\frac{M}{2}}^{\frac{M}{2}-1} x_{w}(m) e^{-j \frac{2 \pi}{M} k\left(m+\frac{1}{2}\right)} \\
& =\frac{A}{2} W(k-v) e^{j \phi}+\frac{A}{2} W(k+v) e^{-j \phi} \\
& +\frac{A_{\mathrm{ih}}}{2} W\left(k-v_{\mathrm{ih}}\right) e^{j \phi_{\mathrm{ih}}}+\frac{A_{\mathrm{ih}}}{2} W\left(k+v_{\mathrm{ih}}\right) e^{-j \phi_{\mathrm{ih}}},
\end{aligned}
$$

where $W(\cdot)$ is the discrete time Fourier transform (DTFT) of the adopted window $w(\cdot)$. For $|\lambda| \ll M$ and $M \gg 1, W(\cdot)$ is given by [12]:

$$
W(\lambda)=\frac{M \sin (\pi \lambda)}{2^{2 H-2} \pi \lambda} \frac{(2 H-2) !}{\prod_{h=1}^{H-1}\left(h^{2}-\lambda^{2}\right)} .
$$

which shows that $W(\cdot)$ exhibits even symmetry.

It is important to note that the second and the fourth terms in (5) represent the contributions of the fundamental spectral image and interharmonic components, respectively.

The inter-bin frequency location estimator returned by the considered IpDFT algorithm is given by [12]:

$$
\hat{\delta}=\frac{(H-1+i) \alpha-H+i}{\alpha+1},
$$

where $i=0$ if $\left|X_{\mathrm{w}}(l-1)\right| \geq\left|X_{\mathrm{w}}(l+1)\right|$ and $i=1$ if $\left|X_{\mathrm{w}}(l-1)\right|$ $<\left|X_{\mathrm{w}}(l+1)\right|$ and $\alpha \stackrel{\text { def }}{=} \frac{\left|X_{\mathrm{w}}(l+i)\right|}{\left|X_{\mathrm{w}}(l-1+i)\right|}$.

The related IpDFT amplitude and phase estimators are given by [11], [12]:

$$
\hat{A}=\frac{2\left|X_{\mathrm{w}}(l)\right|}{|W(-\hat{\delta})|}=\frac{2\left|X_{\mathrm{w}}(l)\right|}{|W(\hat{\delta})|},
$$

and

$$
\hat{\phi}=\measuredangle\left\{X_{\mathrm{w}}(l)\right\},
$$

where $\hat{\delta}$ is the inter-bin frequency location estimate returned by (7). When $l_{\text {ih }} \geq l+1$ and $A_{\text {ih }} \ll A$, due to the interharmonic component, that estimate is affected by the following error [25]: 


$$
\begin{aligned}
& \Delta \delta \cong\left(H+(-1)^{i} \delta\right) \times \\
& {\left[\frac{(-1)^{i} 2 v}{2 v-\delta+(-1)^{i+1} H} \frac{W(2 v-\delta)}{W(\delta)} \cos (2 \phi)+\right.} \\
& \left.\frac{(-1)^{i}\left(v_{\mathrm{ih}}-v\right)}{v_{\mathrm{ih}}-v+\delta+(-1)^{i} H} \frac{A_{\mathrm{ih}}}{A} \frac{W\left(v_{\mathrm{ih}}-v+\delta\right)}{W(\delta)} \cos \left(\phi_{\mathrm{ih}}-\phi\right)\right] .
\end{aligned}
$$

The corresponding relative amplitude and phase estimation errors are given by (see Appendix A):

$$
\begin{aligned}
& \varepsilon_{A} \stackrel{\text { def }}{=} \frac{\hat{A}-A}{A}=\frac{W(2 v-\delta)}{W(\delta)} \cos (2 \phi) \\
& +\frac{A_{\mathrm{ih}}}{A} \frac{W\left(v_{\mathrm{ih}}-v+\delta\right)}{W(\delta)} \cos \left(\phi_{\mathrm{ih}}-\phi\right)-\frac{W^{\prime}(\delta)}{W(\delta)} \Delta \delta,
\end{aligned}
$$

and (see Appendix B):

$$
\begin{gathered}
\Delta \phi \stackrel{\text { def }}{=} \hat{\phi}-\phi=-\frac{W(2 v-\delta)}{W(\delta)} \sin (2 \phi) \\
+\frac{A_{\mathrm{ih}}}{A} \frac{W\left(v_{\mathrm{ih}}-v+\delta\right)}{W(\delta)} \sin \left(\phi_{\mathrm{ih}}-\phi\right),
\end{gathered}
$$

where $W^{\prime}(\delta)$ is the derivative of $W(\cdot)$ with respect to $\delta$ and $\Delta \delta$ is given by (10).

(11) and (12) show that the amplitude error $\varepsilon_{A}$ depends on the frequency error $\Delta \delta$, while the phase error $\Delta \phi$ is independent of it. By using expression (8), after simple calculations, the error $\boldsymbol{\varepsilon}_{A}$ becomes:

$$
\begin{aligned}
& \varepsilon_{A}=\frac{W(2 v-\delta)}{W(\delta)} \cos (2 \phi) \\
& \times\left[\left(H+(-1)^{i} \delta\right) \frac{(-1)^{i+1} 2 v}{2 v-\delta+(-1)^{i+1} H} \frac{W^{\prime}(\delta)}{W(\delta)}+1\right] \\
& +\frac{A_{\mathrm{ih}}}{A} \frac{W\left(v_{\mathrm{ih}}-v+\delta\right)}{W(\delta)} \cos \left(\phi_{\mathrm{ih}}-\phi\right) \\
& \times\left[\left(H+(-1)^{i} \delta\right) \frac{(-1)^{i+1}\left(v_{\mathrm{ih}}-v\right)}{v_{\mathrm{ih}}-v+\delta+(-1)^{i} H} \frac{W^{\prime}(\delta)}{W(\delta)}+1\right] .
\end{aligned}
$$

Observe that both equations (12) and (13) contain two terms that depend on the phases of the fundamental and interharmonic components through a sine function. The first term is the contribution of the spectral interference from the fundamental image component. It decreases as the number of acquired sinewave cycles $v$ increases. The second term is the contribution of

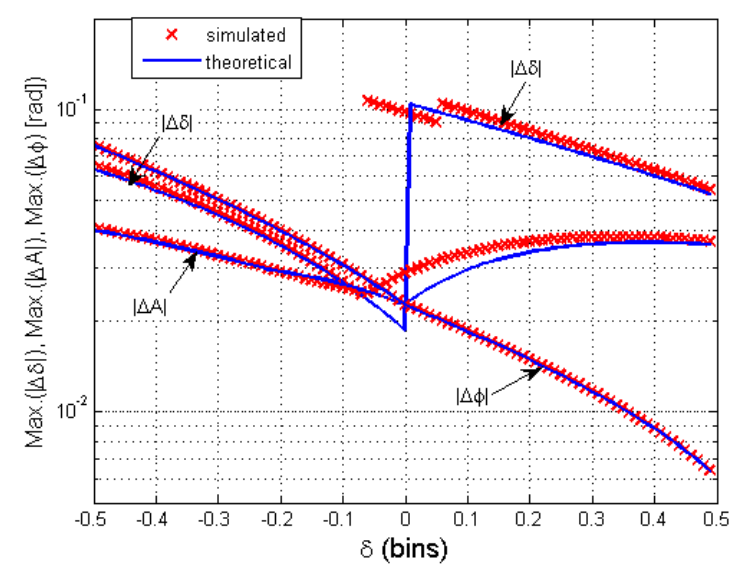

(a) the spectral interference from the interharmonic component. It decreases as the difference between the observed number of interharmonic and fundamental cycles $\nu_{\text {ih }}-v$ increases. Moreover, both errors $\varepsilon_{A}$ and $\Delta \phi$ linearly increase with the relative interharmonic amplitude $A_{\text {ih }} / A$. If the distance $v_{\text {ih }}-v$ is small, and the relative amplitude $A_{\mathrm{ih}} / A$ is quite high, then the contribution of the interharmonic component dominates that of the fundamental image component.

It is also important to note that when acquiring a single signal record, the errors $\Delta \delta, \varepsilon_{A}$ and $\Delta \phi$ exhibit a systematic behaviour since they depend on the constant parameters of the fundamental and the interharmonic components, the window DTFT and its derivative. Unfortunately, the proposed analysis only allows for an evaluation of that contribution (and so providing a type $\mathrm{B}$ method of uncertainty evaluation) when the difference between the recorded number of cycles of the fundamental and the interharmonic components is greater than about three. Indeed, below that threshold, the interference from the interharmonic component is significant, and the IpDFT algorithm does not return accurate parameter estimates, so the derived relationship cannot be effectively applied to evaluate and possibly mitigate - the related systematic contribution.

\section{COMPUTER SIMULATIONS AND EXPERIMENTAL RESULTS}

In this section, both meaningful computer simulations and experimental results are reported in order to check the accuracies of the derived expressions for relative amplitude error (13) and phase error (12) and to verify the correctness of the related conclusions. Furthermore, equation (10) for the inter-bin frequency estimation error is analysed since it affects the amplitude estimation error.

Only simulations and experiments related to specific values of the parameters are reported in the following for the sake of conciseness. However, behaviours very similar to those shown here were obtained in all the considered situations.

\subsection{Simulation results}

The amplitude and the phase of the simulated sine-waves are $A=1$ p.u. and $\phi=\pi / 3 \mathrm{rad}$, respectively. The acquisition length is $M=512$ samples. Each simulation considers 1000 runs in which the initial phase of the interharmonic component $\phi_{\mathrm{h}}$ changes at random in the range $[0,2 \pi) \mathrm{rad}$. The two-term MSD

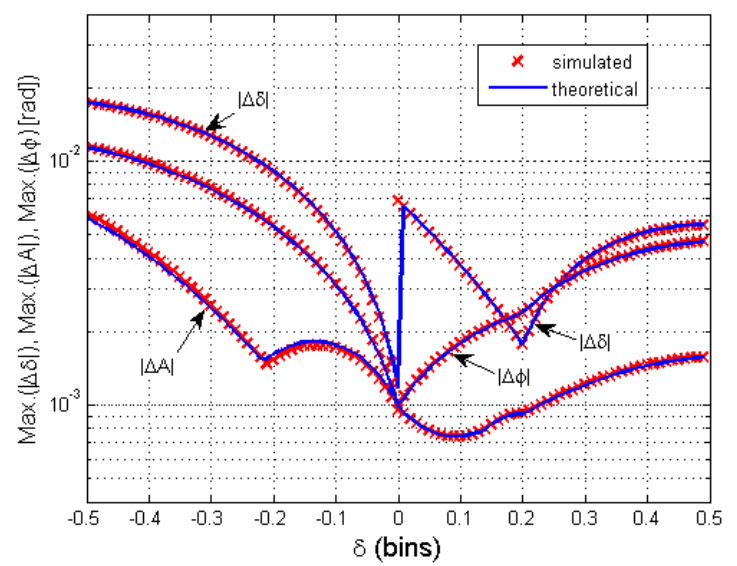

(b)

Figure 1. The simulation and theoretical results related to the maximum values of the error magnitudes $|\Delta \delta|,|\Delta A|$, and $|\Delta \phi|$ versus the inter-bin frequency $\delta$ when $I=2$ cycles, $v_{\text {in }}-v=1.4$ cycles (a) and $v_{\text {ih }}-v=2.8$ cycles (b). $A_{\text {ih }} / A=0.1$ and 1000 runs of $M=512$ samples each are considered. The Hann window is adopted. 
window, known also as Hann window [4], [22], is used.

Figure 1 shows the maximum values of the error magnitudes $|\Delta \delta|,|\Delta A|$, and $|\Delta \phi|$ obtained by simulations and theoretical results as a function of the inter-bin frequency location $\delta$ when $l$ $=2$ cycles, $A_{\text {ih }}=0.1$ p.u., $v_{\text {ih }}-v=1.4$ cycles (Figure $1(\mathrm{a})$ ) and $v_{\text {ih }}-\boldsymbol{v}=2.8$ cycles (Figure $1(\mathrm{~b})$ ).

Figure 1 shows a very good agreement between the simulation and the theoretical results except when $\delta$ is close to zero and $v_{i b}-v$ $=1.4$ cycles. That behaviour is probably due to an incorrect selection of the second interpolation point between the two

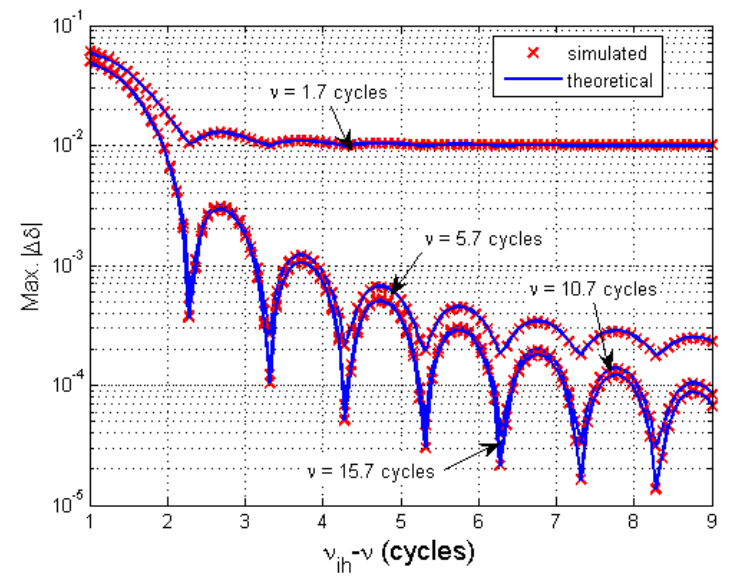

(a)

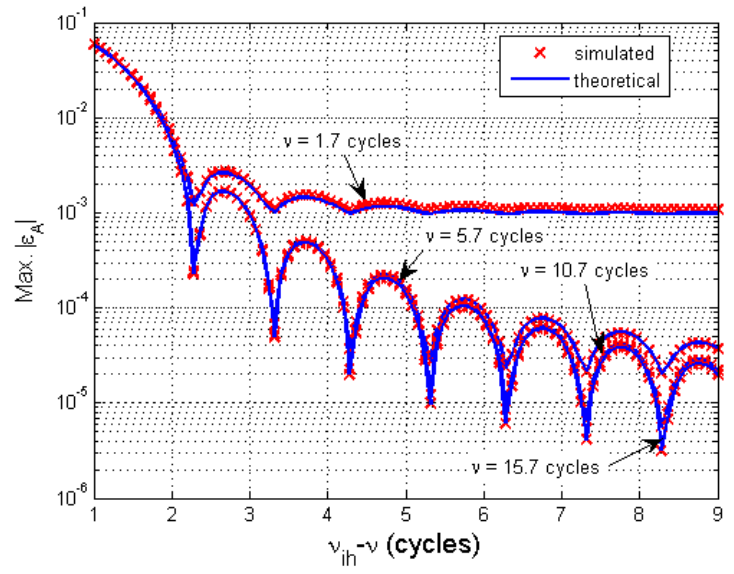

(c)

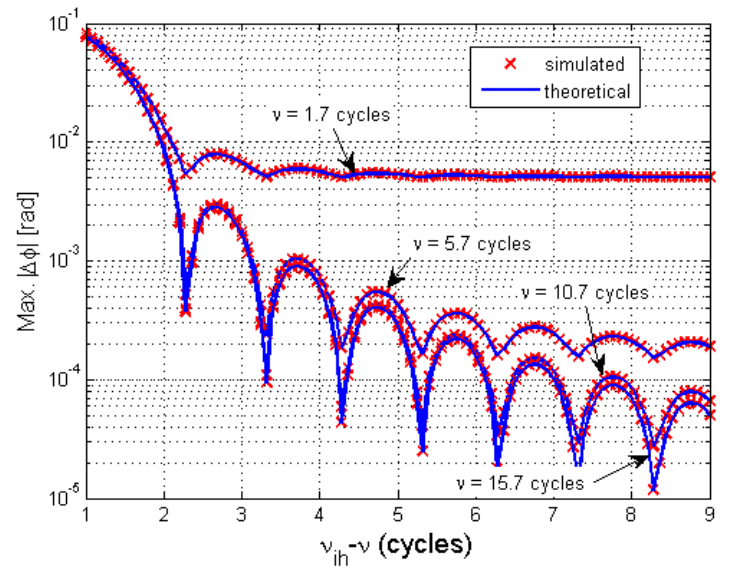

(e) samples $\left|X_{\mathrm{w}}(l-1)\right|$ and $\left|X_{\mathrm{w}}(l+1)\right|$, which exhibit very close values. It is noteworthy that the same behaviour was observed for other values of $v_{\text {ih }}-v<2.4$ cycles. Moreover, this occurred in a range of $\delta$ values that became shorter when $v_{\text {ih }}-v$ increases. Notice also that the simulation and theoretical results related to the phase estimation error are always close each other. Indeed, they do not depend on the estimated inter-bin frequency, see (12).

Figure 2 shows the maximum values of the error magnitudes $|\Delta \delta|,\left|\varepsilon_{A}\right|$, and $|\Delta \phi|$ obtained by the simulation and theoretical

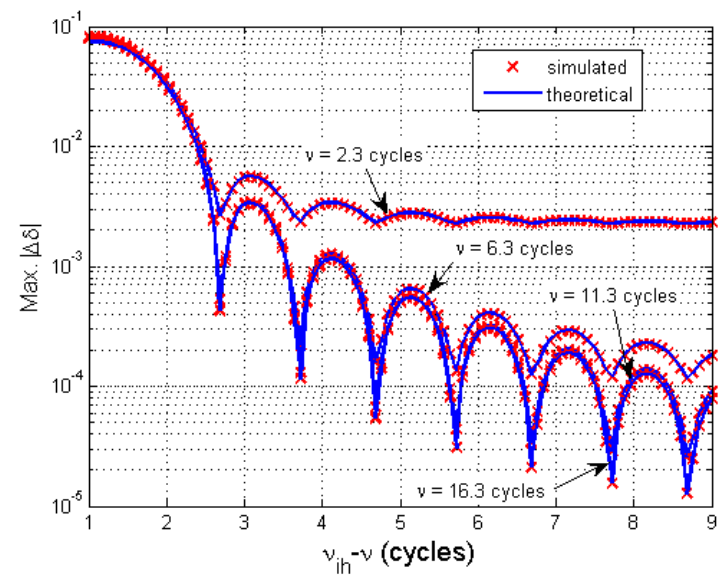

(b)

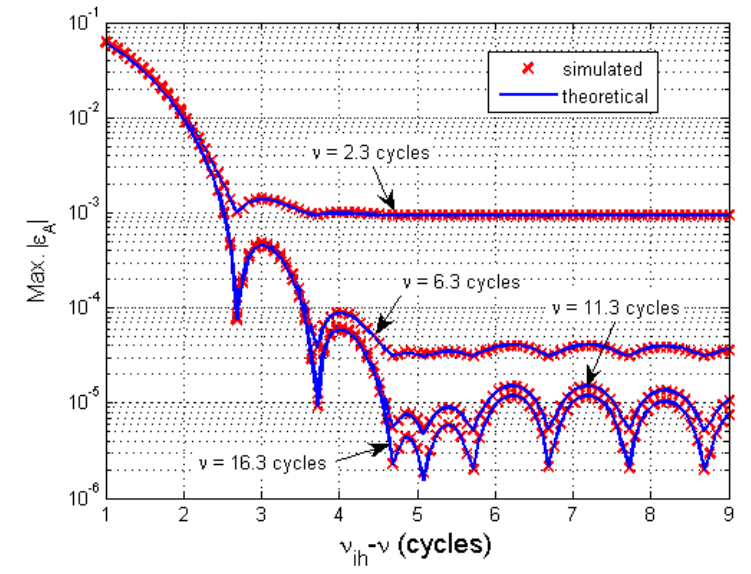

(d)

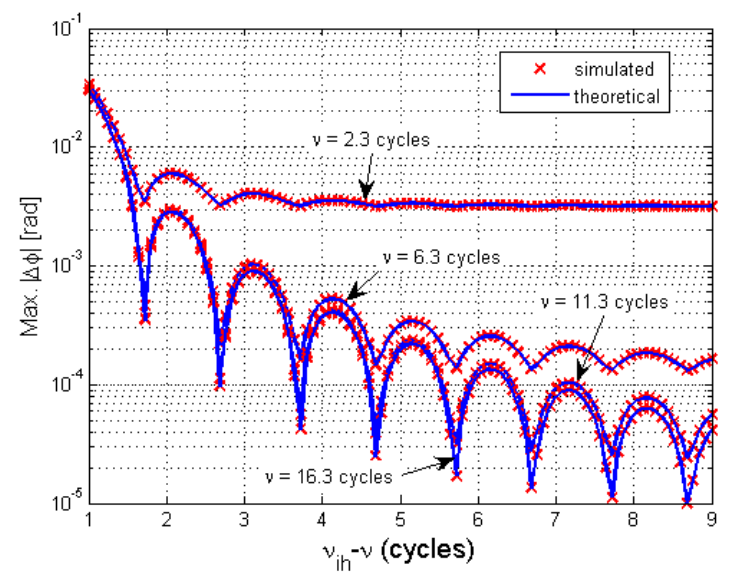

(f)

Figure 2. The simulation and theoretical results related to the maximum values of the error magnitudes $|\Delta \delta|,\left|\varepsilon_{A}\right|$, and $|\Delta \phi|$ versus the distance $v_{\text {in }}-v$ between the observed cycles of the interharmonic and the fundamental components when $v=1.7,5.7,10.7,15.7 \mathrm{cycles}(\mathrm{a})$ and $v=2.3,6.3,11.3,16.3 \mathrm{cycles}(\mathrm{b})$. $A_{\text {ih }} / A=$ 0.1 and 1000 runs of $M=512$ samples each are considered. The Hann window is adopted. 
results versus the difference $v_{\text {ih }}-\nu$ of the observed cycles of the two components when $A_{\text {ih }}=0.1$ p.u., $v=1.7,5.7,10.7,15.7$ cycles (Figure 2(a), (c), (e)) and $v=2.3,6.3,11.3,16.3$ cycles (Figure 2(b), (d), (f)). Notice that Figure 2(a) and (b), (c) and (d), and (e) and (f) are related to the same values of $l$ and $\delta=-0.3$ and $\delta=+0.3$, respectively.

From Figure 2, it follows that there is a very good agreement between the simulation and the theoretical results. Furthermore, it can be observed that all estimation errors are quite significant when the two tones are close to each other (specifically, when $v_{\text {ih }}-v$ is less than about 1.5 cycles) and they mainly depend on the interference due to the interharmonic. Conversely, when the difference $v_{\text {ih }}-v$ increases, the contribution of the fundamental spectral image becomes significant. It is worth noticing that this behaviour holds regardless the value of the number of sine-wave cycles $v$ as soon as it is greater than one cycle, except when $\delta$ is close to zero and the difference $v_{\text {ih }}-v$ is enough high (see Figure 1).

Figure 3 shows the simulation and theoretical results related to the maximum values of the estimation error magnitudes $|\Delta \delta|$,

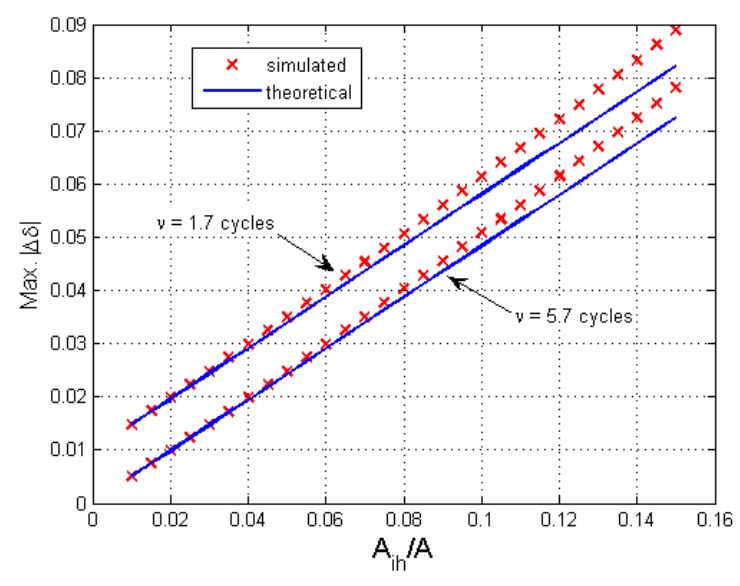

(a)

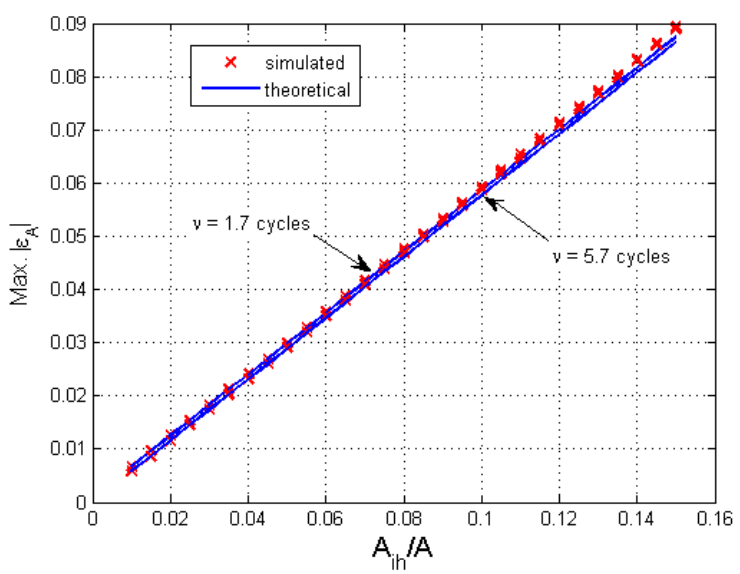

(c)

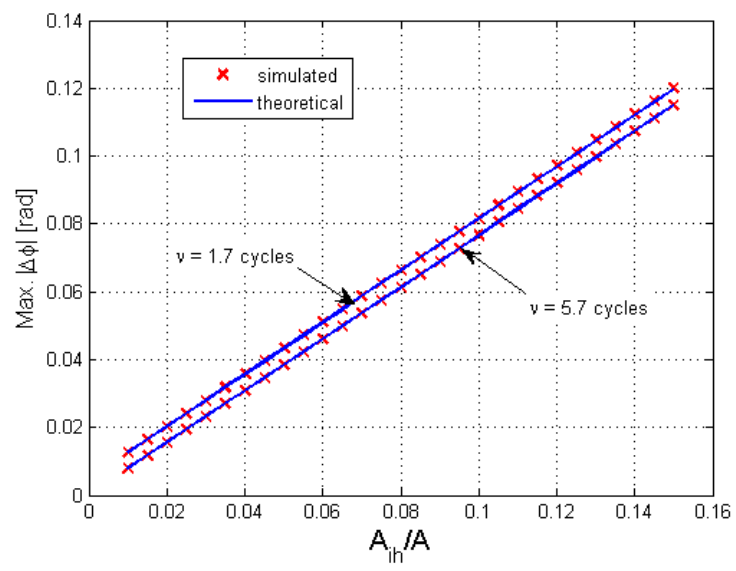

(e)

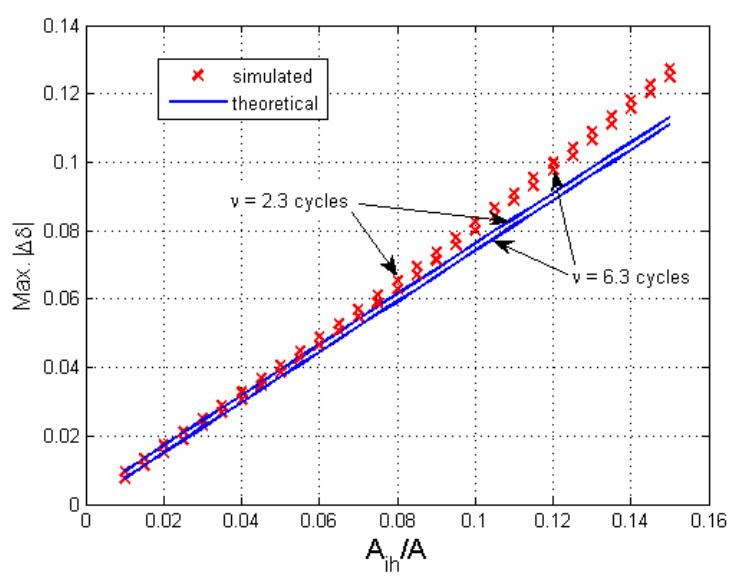

(b)

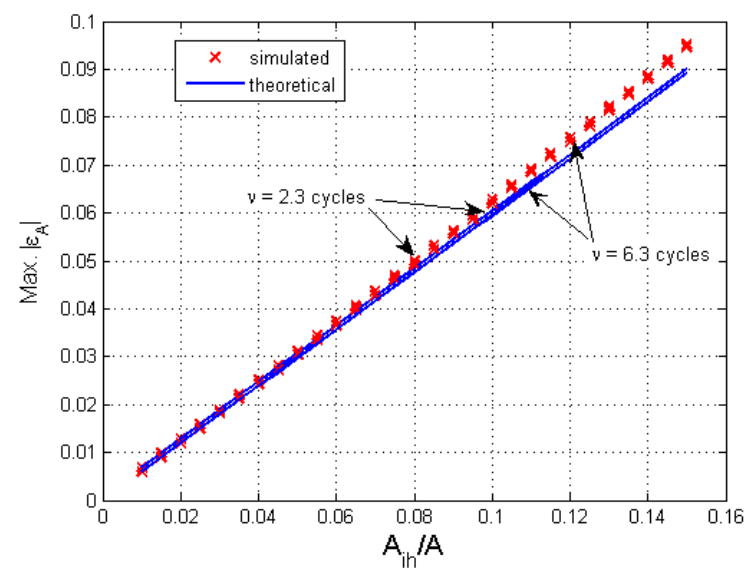

(d)

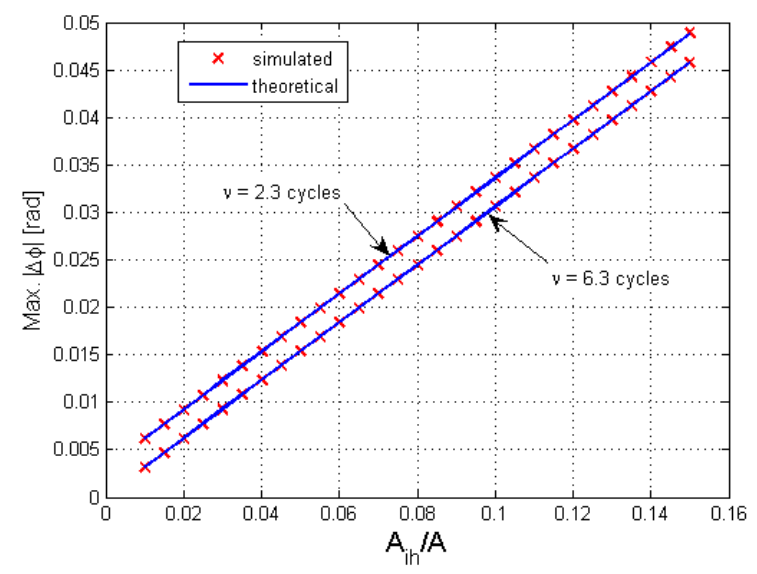

(f)

Figure 3. The simulation and theoretical results related to the maximum values of the error magnitudes $|\Delta \delta|,\left|\varepsilon_{A}\right|$, and $|\Delta \phi|$ versus the ratio $A_{\text {ih }} / A$ when $v=1.7$ and 5.7 cycles (a), and $v=2.3$ and 6.3 cycles (b). $v_{\mathrm{h}}-v=1$ cycle and 1000 runs of $M=512$ samples each are considered. The Hann window is adopted. 
$\left|\varepsilon_{A}\right|$, and $|\Delta \phi|$ as a function of the ratio $A_{\text {ih }} / A$ between interharmonic and fundamental amplitudes, when $v=1.7$ and 5.7 cycles (Figure 3(a), (c), (e)), v $=2.3$ and 6.3 cycles (Figure 3(b), (d), (f)) and $v_{\text {ih }}-v=$ one cycle - the smallest value considered for the difference between the observed cycles of the interharmonic and the fundamental components.

In Figure 3, it can be observed that the simulation and the theoretical results are very close to each other when $A_{\text {in }} / A \leq$
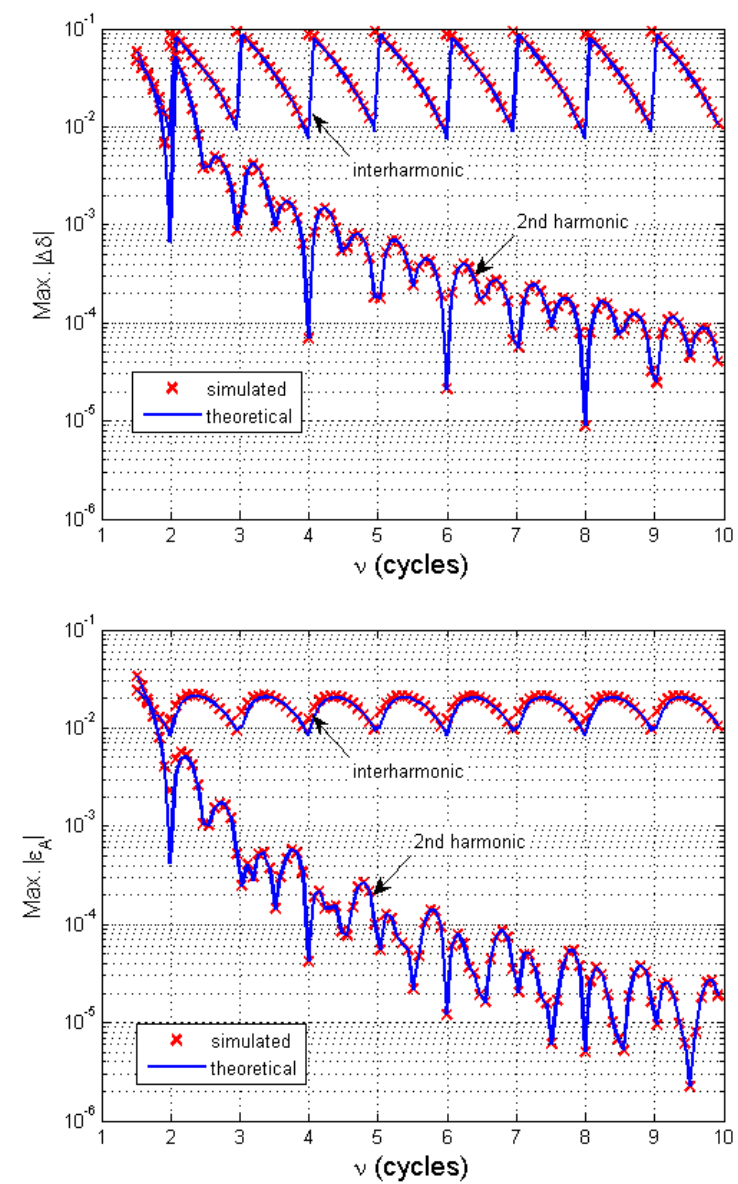

(b)

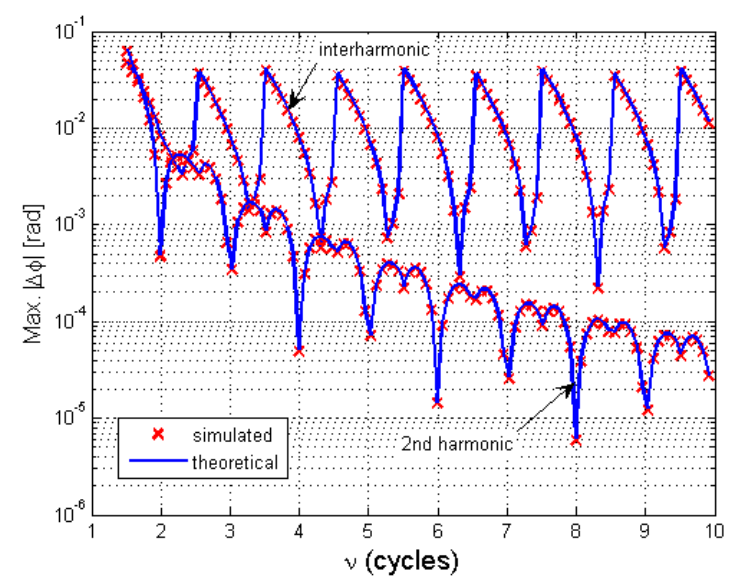

(c)

Figure 4. The simulation and theoretical results related to the maximum values of the error magnitudes $|\Delta \delta|,\left|\varepsilon_{A}\right|$, and $|\Delta \phi|$ versus $v$ when the sine-wave is affected by both an interharmonic and a second harmonic with the same amplitudes, equal to $0.1 \mathrm{p}$.u. The interharmonic is 1.7 cycles from the fundamental component. $A=1$ p.u. and 1000 runs of $M=$ 512 samples each are considered. The Hann window is adopted.
0.1 , except for the phase estimation errors for which there is a very good agreement for all the considered values of $v$ when $A_{\text {ih }} / A<0.65$. This is due to the fact that the expression for the phase error is accurate also when the interharmonic amplitude is much smaller than the fundamental component. Furthermore, all estimation errors linearly increase as the ratio $A_{\text {ih }} / A$ increases, as expected from the theory.

Finally, Figure 4 compares the maximum error magnitudes $|\Delta \delta|,\left|\varepsilon_{A}\right|$, and $|\Delta \phi|$ due to both an interharmonic and a second harmonic as a function of $v, v_{\text {ih }}-v=1.7$ cycles and both the interharmonic and harmonic have the same amplitude equal to 0.1 p.u. The theoretical expressions for the contribution of the second harmonic are obtained from (10), (13), and (12), assuming $v_{\text {ih }}=2 v$.

As we have shown, the sine-wave parameter estimation errors due to the interharmonic are almost independent of the integer part of the number of observed sine-wave cycles, as they mainly end on the inter-bin frequency location $\delta$. Indeed, the second terms in (10), (12), and (13) dominate, and for fixed values of $v_{\text {ih }}-v$, they depend on the inter-bin frequency $\delta$. Therefore, the error magnitude $|\Delta \delta|,\left|\varepsilon_{A}\right|$, and $|\Delta \phi|$ have an almost periodic behaviour with respect to $v$. Conversely, the errors $|\Delta \delta|,\left|\varepsilon_{A}\right|$, and $|\Delta \phi|$ due to the effect of the second harmonic decrease as the number of observed sine-wave cycles $v$ increases. Moreover, the contribution of the interharmonic component dominates, except when $v<2$ cycles, where the contribution due to the fundamental spectral image becomes important.

\subsection{Experimental results}

A block scheme of the experimental setup is shown in Figure 5. The fundamental component was set to an amplitude of $A=$ $2 \mathrm{~V}$ and a frequency of $f_{0}=2 \mathrm{kHz}$, and it was provided at the signal generator OUT1 output, while an interharmonic component of amplitude $A_{\text {ih }}=0.175 \mathrm{~V}$ and frequency $f_{0 \text { ih }}=$ $2.293 \mathrm{kHz}$ were provided at the OUT2 output. The analysed signal, given by the sum of the fundamental and the interharmonic, was obtained at the output of the passive adder. This signal was sampled at a rate of $100 \mathrm{kHz}$ by an NI6023E acquisition board with a full-scale range equal to $10 \mathrm{~V}$. Fifty records of $M=512$ samples each were acquired. Figure 6 shows the experimental and theoretical estimation errors $\Delta \delta, \Delta A$, and $\Delta \phi$ affecting the IpDFT algorithm. The theoretical results were obtained by applying (10), (12), and (13), respectively, in which the involved signal parameters were returned by a linear least squares two-tone fit algorithm. According to that algorithm, the number of the acquired cycles of the fundamental and the interharmonic components, $v$ and $v_{\text {ih }}$ respectively, are firstly determined by (2) and (3), in which $f_{0}$ and $f_{0 \text { ih }}$ are the frequencies of the generated signals. Then, the signal offset and the

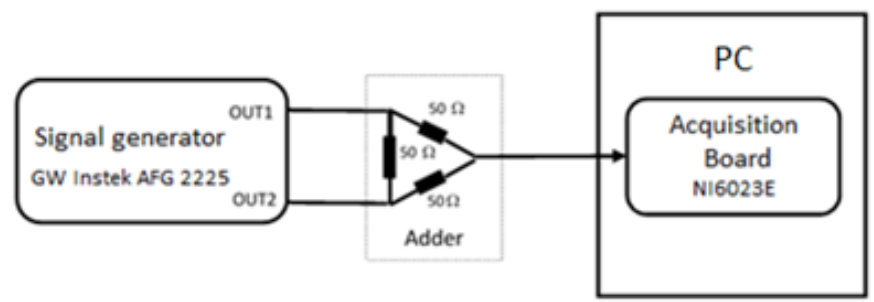

Figure 5. A block scheme of the experimental setup. 
amplitudes and phases of the two components are estimated by applying a linear least squares approach. It is important to note that in the considered situation, the IpDFT algorithm does not return accurate parameter estimates due to the strong interference from the interharmonic component, which abruptly increases as soon as $\left|v_{\text {ih }}-v\right|$ is smaller than about three cycles. It follows that the derived equations (10), (12), and (13) do not allow for an effective compensation for the systematic contribution due to the spectral interference that arises when the IpDFT algorithm is used.
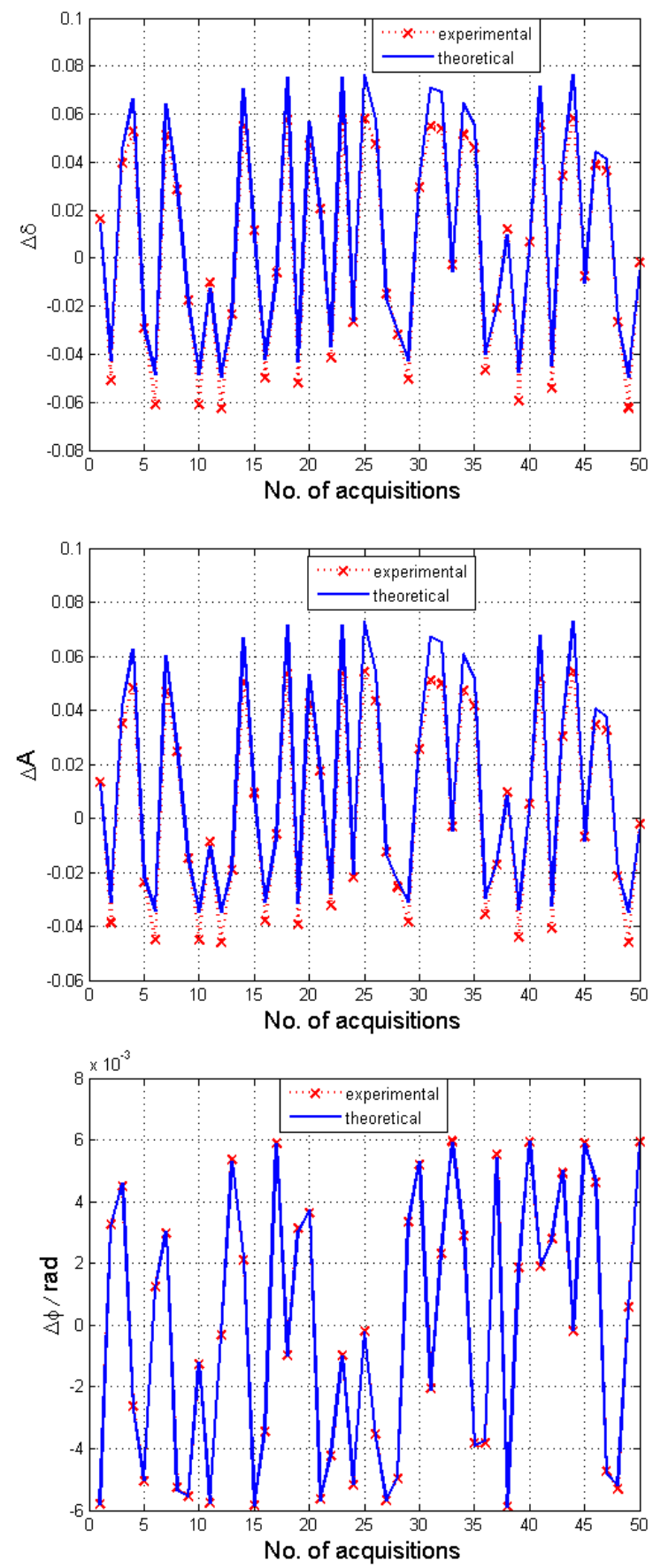

Figure 6. Experimental and theoretical errors $\Delta \delta, \Delta A$, and $\Delta \phi$ affecting the sine-wave parameters IpDFT estimator obtained when considering 50 records of $M=512$ samples each. Real sine-waves affected by an interharmonic are separated by about 1.5 cycles. Theoretical results are achieved by using the linear least squares two-tone fit algorithm.
Figure 6 shows that the experimental and theoretical results for the errors $\Delta \delta$ and $\Delta A$ are close to each other, while those related to the error $\Delta \phi$ almost coincide, thereby validating the performed analysis.

\section{CONCLUSIONS}

The analytical expressions for the errors that affect the sinewave amplitude and phase estimates returned by the classical IpDFT algorithm based on a generic MSD window have been derived in the case when the sine-wave is corrupted by a smallamplitude interharmonic located at least one bin apart the unknown frequency. The derived expressions show that the amplitude error depends on the inter-bin frequency location error, while the estimation of the phase referred at the centre of the observation interval is independent of the sine-wave frequency error. Both expressions for the amplitude and phase errors contain two terms that exhibit sine-like behaviour. One term represents the contribution of the spectral interference from the fundamental image component, while the other one is the contribution of the spectral interference from the interharmonic component. In particular, the obtained expressions show when the contribution due to an interharmonic with a frequency located closely to the sine-wave one can dominate the detrimental effect of the second harmonic.

Both computer simulations and experimental results confirm the accuracies of the derived expressions.

\section{APPENDIX A}

\section{Derivation of the expression for the relative amplitude error $\varepsilon_{A}$}

When $l_{\text {in }} \geq l+1$ and $A_{\text {ih }} \ll A$, we have [25]:

$$
\begin{aligned}
& \left|X_{\mathrm{w}}(l)\right| \cong \frac{A}{2} W(\delta)+\frac{A}{2} W(2 v-\delta) \cos (2 \phi) \\
& +\frac{A_{\mathrm{ih}}}{2} W\left(v_{\mathrm{ih}}-v+\delta\right) \cos \left(\phi_{\mathrm{ih}}-\phi\right) .
\end{aligned}
$$

Since $\hat{\delta}=\delta+\Delta \delta$, with $|\Delta \delta| \ll|\delta|, W(\hat{\delta})$ can be accurately expressed by a first-order Taylor's series about $\delta$ :

$$
W(\hat{\delta}) \cong W(\delta)+W^{\prime}(\delta) \Delta \delta,
$$

where $W^{\prime}(\delta)$ is the derivative of $W(\cdot)$ with respect to $\delta$.

By using expressions (14) and (15) in (8), the estimator $\hat{A}$ becomes:

$$
\begin{aligned}
& \hat{A} \cong {\left[A+A \frac{W(2 v-\delta)}{W(\delta)} \cos (2 \phi)\right.} \\
&+\left.A_{\mathrm{ih}} \frac{W\left(v_{\mathrm{ih}}-v+\delta\right)}{W(\delta)} \cos \left(\phi_{\mathrm{ih}}-\phi\right)\right] \\
& \times {\left[1+\frac{W^{\prime}(\delta)}{W(\delta)} \Delta \delta\right]^{-1} . } \\
& \text { Since }\left|\frac{W^{\prime}(\delta)}{W(\delta)} \Delta \delta\right| \ll 1, \text { using the app } \\
& 1-x, \text { when }|x| \ll 1,(\mathrm{~A} .3) \text { becomes: } \\
& \hat{A} \cong\left[A+A \frac{W(2 v-\delta)}{W(\delta)} \cos (2 \phi)\right. \\
&\left.+A_{i h} \frac{W\left(v_{\mathrm{ih}}-v+\delta\right)}{W(\delta)} \cos \left(\phi_{\mathrm{ih}}-\phi\right)\right]
\end{aligned}
$$$$
\text { Since }\left|\frac{W^{\prime}(\delta)}{W(\delta)} \Delta \delta\right| \ll 1 \text {, using the approximation }(1+x)^{-1} \cong
$$ 


$$
\times\left[1-\frac{W^{\prime}(\delta)}{W(\delta)} \Delta \delta\right]
$$

The second and third terms in the first square brackets of (17) become negligible when multiplied by $\frac{W^{\prime}(\delta)}{W(\delta)} \Delta \delta$. Thus, (17) becomes:

$$
\begin{gathered}
\hat{A} \cong A+A \frac{W(2 v-\delta)}{W(\delta)} \cos (2 \phi)+ \\
+A_{i h} \frac{W\left(v-v_{\mathrm{ih}}-\delta\right)}{W(\delta)} \cos \left(\phi_{\mathrm{ih}}-\phi\right) \\
-A \frac{W^{\prime}(\delta)}{W(\delta)} \Delta \delta .
\end{gathered}
$$

From (18), the amplitude estimation error results in:

$$
\begin{aligned}
& \Delta A \stackrel{\text { def }}{=} \hat{A}-\mathrm{A} \cong A \frac{W(2 v-\delta)}{W(\delta)} \cos (2 \phi)+ \\
& +A_{\mathrm{ih}} \frac{W\left(v_{\mathrm{ih}}-v+\delta\right)}{W(\delta)} \cos \left(\phi_{\mathrm{ih}}-\phi\right)-A \frac{W^{\prime}(\delta)}{W(\delta)} \Delta \delta .
\end{aligned}
$$

Finally, by using (19), equation (11) can be easily obtained.

\section{APPENDIX B}

\section{Derivation of the expression for the phase error $\Delta \phi$}

From (7), the expression of $X_{\mathrm{w}}(\zeta)$ is given by:

$$
\begin{aligned}
& X_{\mathrm{w}}(l)=\frac{A}{2}[W(\delta)+W(2 v-\delta)] \cos (\phi) \\
& +\frac{A_{\mathrm{ih}}}{2}\left[W\left(v_{\mathrm{ih}}-v+\delta\right)+W\left(v_{\mathrm{ih}}+v-\delta\right)\right] \cos \left(\phi_{\mathrm{ih}}\right) \\
& +j \frac{A}{2}[W(\delta)-W(2 v-\delta)] \sin (\phi) \\
& +j \frac{A_{\mathrm{ih}}}{2}\left[W\left(v_{\mathrm{ih}}-v+\delta\right)-W\left(v_{\mathrm{ih}}+v-\delta\right)\right] \sin \left(\phi_{\mathrm{ih}}\right)
\end{aligned}
$$

By using (20), observing that the contribution of $W\left(v_{\text {ih }}+v\right.$ $\delta$ ) can be neglected compared with the other terms, after some simple calculations, equation (9) becomes:

$$
\begin{aligned}
& \hat{\phi} \cong \tan ^{-1}\left\{\operatorname { t a n } ( \phi ) \left[1-\frac{W(2 v-\delta)}{W(\delta)}\right.\right. \\
& \left.+\frac{A_{\mathrm{ih}}}{A} \frac{W\left(v_{\mathrm{ih}}-v+\delta\right)}{W(\delta)} \frac{\sin \left(\phi_{\mathrm{ih}}\right)}{\sin (\phi)}\right] \\
& \times\left[1+\frac{W(2 v-\delta)}{W(\delta)}\right. \\
& \left.\left.+\frac{A_{\mathrm{ih}}}{A} \frac{W\left(v_{\mathrm{ih}}-v+\delta\right)}{W(\delta)} \frac{\cos \left(\phi_{\mathrm{ih}}\right)}{\cos (\phi)}\right]^{-1}\right\} .
\end{aligned}
$$

Observing that $\left|\frac{W(2 v-\delta)}{W(\delta)}+\frac{A_{\mathrm{ih}}}{A} \frac{W\left(v_{\mathrm{ih}}-v+\delta\right)}{W(\delta)} \frac{\cos \left(\phi_{\mathrm{ih}}\right)}{\cos (\phi)}\right| \ll 1$ and using the approximation $(1+x)^{-1} \cong 1-x$, when $|x| \ll 1$, (21) becomes:

$$
\begin{aligned}
& \hat{\phi} \cong \tan ^{-1}\left\{\operatorname { t a n } ( \phi ) \left[1-\frac{W(2 v-\delta)}{W(\delta)}\right.\right. \\
& \left.+\frac{A_{\mathrm{ih}}}{A} \frac{W\left(v_{\mathrm{ih}}-v+\delta\right)}{W(\delta)} \frac{\sin \left(\phi_{\mathrm{ih}}\right)}{\sin (\phi)}\right] \times \\
& \left.\left[1-\frac{W(2 v-\delta)}{W(\delta)}-\frac{A_{\mathrm{ih}}}{A} \frac{W\left(v_{\mathrm{ih}}-v+\delta\right)}{W(\delta)} \frac{\cos \left(\phi_{\mathrm{ih}}\right)}{\cos (\phi)}\right]\right\} .
\end{aligned}
$$

The second and third terms in the first square brackets of (22) multiplied by $\left[\frac{W(2 v-\delta)}{W(\delta)}+\frac{A_{\mathrm{ih}}}{A} \frac{W\left(v_{\mathrm{ih}}-v+\delta\right)}{W(\delta)} \frac{\cos \left(\phi_{\mathrm{ih}}\right)}{\cos (\phi)}\right]$ are very small compared with the other terms so that they can be neglected. Thus, after some calculations, the estimator $\hat{\phi}$ becomes:

$$
\begin{aligned}
& \hat{\phi} \cong \tan ^{-1}\left\{\tan (\phi)-\frac{2 W(2 v-\delta)}{W(\delta)} \tan (\phi)\right. \\
& \left.+\frac{A_{\mathrm{ih}}}{A} \frac{W\left(v_{\mathrm{ih}}-v+\delta\right)}{W(\delta)} \frac{\sin \left(\phi_{\mathrm{ih}}-\phi\right)}{\cos ^{2}(\phi)}\right\} .
\end{aligned}
$$

By expressing the $\tan ^{-1}(\cdot)$ function using its first-order Taylor's series about $\tan (\phi)$, the following expression for $\hat{\phi}$ is achieved:

$$
\begin{aligned}
& \hat{\phi} \cong \phi-\frac{W(2 v-\delta)}{W(\delta)} \sin (\phi) \\
& +\frac{A_{\mathrm{ih}}}{A} \frac{W\left(v_{\mathrm{ih}}-v+\delta\right)}{W(\delta)} \sin \left(\phi_{i h}-\phi\right) .
\end{aligned}
$$

Finally, using (24), equation (12) can be easily obtained.

\section{REFERENCES}

[1] S. M. Kay, Fundamentals of Statistical Signal Processing: Estimation Theory, Prentice-Hall, Upper Saddle River, NJ, 1993.

[2] P. Stoica, R. L. Moses, Introduction to Spectral Analysis, vol. 1, Prentice-Hall, Englewood Cliffs, NJ, 1997.

[3] S. L. Marple, Digital Spectral Analysis, Prentice-Hall, Englewood Cliffs, 1987.

[4] F. J. Harris, On the use of windows for harmonic analysis with the discrete Fourier transform, Proc. of the IEEE 66, 1978, pp. 51-83. DOI: https://doi.org/10.1109/PROC.1978.10837

[5] C. Offelli, D. Petri, Interpolation techniques for real-time multifrequency analysis, IEEE Trans. Instrum. Meas. 39 (1990) pp. 106-111.

DOI: https://doi.org/10.1109/IMTC.1989.36879

[6] B. G. Quinn, Estimating frequency by interpolation using Fourier coefficients, IEEE Trans. Signal Process. 42 (1994) pp. 1264-1268. DOI: https://doi.org/10.1109/78.295186

[7] B. G. Quinn, Estimation of frequency, amplitude, and phase from the DFT of a time series, IEEE Trans. Signal Process. 45 (1997) pp. 814-817.

DOI: https://doi.org/10.1109/78.558515

[8] D. C. Rife, G. A. Vincent, Use of the discrete Fourier transform in the measurement of frequencies and levels of tones, Bell Syst. Tech. J., 49 (1970) pp. 197-228.

DOI: https://doi.org/10.1002/j.1538-7305.1970.tb01766.x

[9] T. Grandke, Interpolation algorithms for discrete Fourier transforms of weighted signals, IEEE Trans. Instrum. Meas. 32 (1983) pp. 350-355.

DOI: https://doi.org/10.1109/TIM.1983.4315077

[10] J. Schoukens, R. Pintelon, H. Van Hamme, The interpolated fast Fourier transform: a comparative study, IEEE Trans. Instrum. Meas. 41 (1992) pp. 226-232.

DOI: https://doi.org/10.1109/19.137352

[11] C. Offelli, D. Petri, The influence of windowing on the accuracy of multifrequency signal parameter estimation, IEEE Trans. Instrum. Meas. 41 (1992) pp. 256-261.

DOI: https://doi.org/10.1109/19.137357

[12] D. Belega, D. Dallet, Multifrequency signal analysis by interpolated DFT method with maximum sidelobe decay windows, Measurement 42 (2009) pp. 420-426.

DOI: https://doi.org/10.1016/j.measurement.2008.08.006

[13] D. Belega, D. Dallet, D. Petri, Statistical description of the sinewave frequency estimator provided by the interpolated DFT 
method, Measurement 45 (2012) pp. 109-117.

DOI: https://doi.org/10.1016/i.measurement.2011.09.010

[14] K. Duda, DTF interpolation algorithm for Kaiser-Bessel and Dolph-Chebyshev windows, IEEE Trans. Instrum. Meas. 60 (2011) pp. 784-790.

DOI: https://doi.org/10.1109/TIM.2010.2046594

[15] D. Agrež, Dynamics of the frequency estimation in the frequency domain, IEEE Trans. Instrum. Meas. 56 (2007) pp. 2111-2118. DOI: https://doi.org/10.1109/IMTC.2004.1351218

[16] D. Agrež, Weighted multipoint interpolated DFT to improve amplitude estimation of multifrequency signal, IEEE Trans. Instrum. Meas. 51 (2002) pp. 287-292.

DOI: https://doi.org/10.1109/19.997826

[17] D. Belega, D. Dallet, D. Petri, Accuracy of sine-wave frequency estimation by multipoint interpolated DFT approach, IEEE Trans. Instrum. Meas. 59 (2010) pp. 2808-2815. DOI: https://doi.org/10.1109/TIM.2010.2060870

[18] K. F. Chen, J. T. Jiang, S. Crowsen, Against the long-range spectral leakage of the cosine window family, Comput. Phys. Commun. 180 (2009) pp. 904-911.

DOI: https://doi.org/10.1016/i.cpc.2008.12.019

[19] K. F. Chen, X. Cao, Y. F. Li, Sine wave fitting to short records initialized with the frequency retrieved from Hanning windowed FFT spectrum, Measurement 42 (2009) pp. 127-135. DOI: https://doi.org/10.1016/i.measurement.2008.04.007

[20] J. Borkowski, D. Kania, J. Mroczka, Interpolated-DFT-based fast and accurate frequency estimation for the control of power, IEEE
Trans. Ind. Electron. 61 (2014) pp. 7026-7034.

DOI: https://doi.org/10.1109/TIE.2014.2316225

[21] Y. Wang, W. Wei, J. Xiang, Multipoint interpolated DFT for sine waves in short records with DC components, Signal Process. 131 (2017) pp. 161-170.

DOI: https://doi.org/10.1016/i.sigpro.2016.08.013

[22] A. H. Nuttall, Some windows with very good sidelobe behavior, IEEE Trans. Acoust. Speech Signal Process. ASSP-29 (1981) pp. 84-91.

DOI: https://doi.org/10.1109/TASSP.1981.1163506

[23] D. Belega, D. Petri, D. Dallet, Impact of harmonics on the interpolated DFT frequency estimator, Mech. Syst. Signal Process. 66-67 (2016) pp. 349-360.

DOI: https://doi.org/10.1016/i.ymssp.2015.05.025

[24] D. Belega, D. Petri, Accuracy of the synchrophasor estimator returned by the interpolated DFT algorithm under off-nominal frequency and harmonic conditions, Proc. of the IEEE Applied Measurements for Power Systems (AMPS) Workshop, Bologna, Italy, 26-28 September 2018, pp. 105-110. DOI: https://doi.org/10.1109/AMPS.2018.8494892

[25] D. Belega, D. Petri, D. Dallet, Contribution of interharmonic component on the interpolated DFT frequency estimator, Proc. of the 24th IMEKO TC4 International Symposium, Xi'an, China, 17-20 September 2019. https://www.imeko.org/publications/tc4-2019/IMEKO-TC42019-034.pdf 